\title{
Pinched exponential volume growth implies an infinite dimensional isoperimetric inequality
}

\author{
Itai Benjamini Oded Schramm
}

\begin{abstract}
Let $G$ be a graph which satisfies $c^{-1} a^{r} \leq|B(v, r)| \leq c a^{r}$, for some constants $c, a>1$, every vertex $v$ and every radius $r$. We prove that this implies the isoperimetric inequality $|\partial A| \geq C|A| / \log (2+|A|)$ for some constant $C=C(a, c)$ and every finite set of vertices $A$.
\end{abstract}

A graph $G=(V(G), E(G))$ has pinched growth $f(r)$ if there are two constants $0<c<C<\infty$ so that every ball $B(v, r)$ of radius $r$ centered around a vertex $v \in V(G)$ satisfies

$$
c f(r)<|B(v, r)|<C f(r) .
$$

For example, Cayley graphs and vertex-transitive graphs have pinched growth.

It is easy to come up with an example of a tree for which every ball satisfies $|B(v, r)| \geq 2^{r / 2}$, yet there are arbitrarily large finite subsets of $G$ with one boundary vertex. For example, start with $\mathbb{N}$ (an infinite one-sided path) and connect every vertex $n$ to the root of a binary tree of depth $n$. This tree does not have pinched growth.

We will see that, perhaps surprisingly, the additional assumption of pinched exponential growth (that is, pinched growth $a^{r}$, for some $a>1$ ) implies an infinite dimensional isoperimetric inequality. For a set $A \subset V(G)$ of vertices denote by $\partial A$ the (vertex) boundary of $A$, consisting of vertices outside of $A$ which have a neighbor in $A$.

Theorem 1. Let $G$ be an infinite graph with pinched growth $a^{r}$, where $a>1$. Then there is a constant $c>0$ such that for every finite set of vertices $A \subset V(G)$,

$$
|\partial A| \geq c|A| / \log (2+|A|) .
$$


We say that $G$ satisfies an $s$-dimensional isoperimetric inequality if there is a $c>0$ such that $|\partial A| \geq c|A|^{(s-1) / s}$ holds for every finite $A \subset V(G)$. Thus, (11) may be considered an infinite dimensional isoperimetric inequality.

Coulhon and Saloff-Coste [4 proved that when $G$ is a Cayley graph of an infinite, finitely-generated, group, the isoperimetric inequality

$$
|\partial A| \geq \frac{|A|}{4 m \phi(2|A|)}
$$

holds for every finite $A \subset V(G)$, where $m$ is the number of neighbors every vertex has and $\phi(n)=\inf \{r \geq 1:|B(v, r)| \geq n\}$ (here, $v \in V(G)$ is arbitrary). This result implies Theorem 1 for the case where $G$ is a Cayley graph, even when the upper bound in the pinched growth condition is dropped. The tree example discussed above shows that Theorem 1 is not valid without the upper bound. Thus, the (short and elegant) proof of (2) from [4] does not generalize to give Theorem 11. and, in fact, the proof below does not seem related to the arguments from [4].

It is worthwhile to note that (2) is also interesting for Cayley graphs with sub-exponential growth. For example, it shows that $\mathbb{Z}^{d}$ satisfies a $d$ dimensional isoperimetric inequality.

Another related result, with some remote similarity in the proof, is due to Babai and Szegedy [1. They prove that for a finite vertex transitive graph $G$, and $A \subset V(G), 0<|A|<|G| / 2$,

$$
|\partial A| \geq|A| /(1+\operatorname{diam} G) \text {. }
$$

The isoperimetric inequality (11) is sharp up to the constant, since there are groups with pinched growth $a^{r}$ where (11) cannot be improved. Examples include the lamplighter on $\mathbb{Z}[7]$. See $[6]$ for a discussion of growth rates of groups and many related open problems.

Regarding pinched polynomial growth, it is known that for every $d>1$ there is a tree with pinched growth $r^{d}$ containing arbitrarily large sets $A$ with $|\partial A|=1$, see, e.g., 3 .

Problem 1. Does every graph of a pinched exponential growth contain a tree with pinched exponential growth?

In 2] it was shown that every graph satisfying the linear isoperimetric inequality $|\partial A| \geq c|A|(c>0)$ contains a tree satisfying such an inequality, possibly with a different constant. The question whether one can find a 
spanning tree with a linear isoperimetric inequality was asked earlier [5]. It follows from Theorem 1 that a tree with pinched exponential growth satisfies the linear isoperimetric inequality. (If a tree satisfies $|\partial A| \geq 3$ for every vertex set $A$ of size at least $k$, then every path of $k$ vertices in the tree must contain a branch point, a point whose removal will give at least 3 infinite components. Consequently the tree contains a modified infinite binary tree, where every edge is subdivided into at most $k$ edges.) Consequently, Problem 1 is equivalent to the question whether every graph with pinched exponential growth contains a tree satisfying a linear isoperimeteric inequality.

As a warm up for the proof of Theorem 11, here is an easy argument showing that when $G$ has pinched growth $a^{r}$ it satisfies a two-dimensional isoperimetric inequality. Let $A \subset V(G)$ be finite. Let $v$ be a vertex of A that is farthest from $\partial A$, and let $r$ be the distance from $v$ to $\partial A$. Note that $B(v, 2 r) \subset \bigcup_{u \in \partial A} B(u, r)$. This gives, $a^{2 r} \leq O(1)|\partial A| a^{r}$, and therefore $O(1)|\partial A| \geq a^{r}$. On the other hand, $\bigcup_{u \in \partial A} B(u, r) \supset A$, which gives $O(1)|\partial A| a^{r} \geq|A|$. Hence, $O(1)|\partial A|^{2} \geq|A|$.

Proof of Theorem. For vertices $v, u$ set $z(v, u):=a^{-d(v, u)}$, where $d(v, u)$ is the graph distance between $v$ and $u$ in $G$.

We estimate in two ways the quantity

$$
Z=Z_{A}:=\sum_{v \in A} \sum_{u \in \partial A} z(v, u)
$$

Fix $v \in A$. For every $w \notin A$, fix some geodesic path from $v$ to $w$, and let $w^{\prime}$ be the first vertex in $\partial A$ on this path. Let $R$ be sufficiently large so that $|B(v, R)| \geq 2|A|$, and set $W:=B(v, R) \backslash A$. Then

$$
\left|\left\{\left(w, w^{\prime}\right): w \in W\right\}\right|=|W| \geq a^{R} / O(1) .
$$

On the other hand, we may estimate the left hand side by considering all possible $u \in \partial A$ as candidates for $w^{\prime}$. If $w \in W$, then $d\left(v, w^{\prime}\right)+d\left(w^{\prime}, w\right) \leq R$. Thus, each $u$ is equal to $w^{\prime}$ for at most $O(1) a^{R-d(v, u)}$ vertices $w \in W$. This gives

$$
\left|\left\{\left(w, w^{\prime}\right): w \in W\right\}\right| \leq O(1) \sum_{u \in \partial A} a^{R-d(u, v)}=O(1) a^{R} \sum_{u \in \partial A} z(v, u) .
$$

Combining these two estimates yields $O(1) \sum_{u \in \partial A} z(v, u) \geq 1$. By summing over $v$, this implies

$$
O(1) Z \geq|A| \text {. }
$$


Now fix $u \in \partial A$, set $m_{r}:=|\{v \in A: d(v, u)=r\}|$, and consider

$$
Z(u):=\sum_{v \in A} z(v, u)=\sum_{r} m_{r} a^{-r}
$$

For $r \leq \log |A| / \log a$, we use the inequality $m_{r} \leq|B(u, r)|=O(1) a^{r}$, while for $r>\log |A| / \log a$, we use $m_{r} \leq|A|$. We apply these estimates to (41), and get $Z(u) \leq O(1) \log (2+|A|)$, which gives $Z=\sum_{u \in \partial A} Z(u) \leq$ $O(1)|\partial A| \log (2+|A|)$. Together with (3) , this gives (11).

Next, we present a slightly different version of Theorem 11, which also applies to finite graphs.

Theorem 2. Let $G$ be a finite or infinite graph, $c>0, a>1, R \in \mathbb{N}$, and suppose that $c^{-1} a^{r} \leq|B(v, r)| \leq c a^{r}$ holds for all $r=1,2, \ldots, R$ and for all $v \in V(G)$. Then there is a constant $C=C(a, c)$, depending only on a and $c$, such that

$$
C|\partial A| \geq|A| / \log (2+|A|)
$$

holds for every finite $A \subset V(G)$ with $|A| \leq C^{-1} a^{R}$.

The proof is the same. A careful inspection of the proof shows that one only needs the inequality $c^{-1} a^{r} \leq|B(v, r)|$ to be valid for $v \in A$ and the inequality $|B(v, r)| \leq c a^{r}$ only for $v \in \partial A$.

Acknowledgements: We thank Thierry Coulhon and Iftach Haitner for useful disscusions.

\section{References}

[1] L. Babai and M. Szegedy, Local expansion of symmetrical graphs, Combin. Probab. Comput. 1 (1992), no. 1, 1-11.

[2] I. Benjamini and O. Schramm, Every graph with a positive Cheeger constant contains a tree with a positive Cheeger constant. Geom. Funct. Anal. vol. 7 (1997), no. 3, 403-419.

[3] I. Benjamini and O. Schramm, Recurrence of distributional limits of finite planar graphs. Electron. J. Probab. 6 (2001), no. 23, 13 pp. (electronic). 
[4] T. Coulhon and L. Saloff-Coste, Isopérimétrie pour les groupes et les variétés. (French) [Isoperimetry for groups and manifolds] Rev. Mat. Iberoamericana 9 (1993), no. 2, 293-314.

[5] W. Deuber, M. Simonovits and V. Sós, A note on paradoxical metric spaces. Studia Sci. Math. Hungar. 30 (1995), no. 1-2, 17-23.

[6] P. de la Harpe, Topics in geometric group theory. Chicago Lectures in Mathematics. University of Chicago Press, Chicago, IL, 2000. vi+310 pp.

[7] R. Lyons, R. Pemantle and Y. Peres, Random walks on the lamplighter group. Ann. Probab. 24 (1996), no. 4, 1993-2006. 